\title{
Acceptance of preventive surgeries by Israeli women who had undergone BRCA testing
}

\author{
Vardit Kram · Tamar Perez $\cdot$ Michal Sagi
}

Published online: 12 January 2007

(C) Springer Science+Business Media B.V. 2007

Familial Cancer (2006)

DOI 10.1007/s10689-006-0002-z

Correction:

\begin{tabular}{lllr}
\hline $\begin{array}{l}\text { Page } \\
\text { number }\end{array}$ & $\begin{array}{l}\text { Table } \\
\text { number }\end{array}$ & Incorrect & Correct \\
\hline 3 & 1 & N $43(43.4 \%)$ & N $56(56.6 \%)$ \\
& & $56(56.6 \%)$ N.S. & $43(43.4 \%)$ \\
\hline
\end{tabular}

The online version of the original article can be found at http:// dx.doi.org/10.1007/s10689-006-0002-z

V. Kram $\cdot$ M. Sagi $(\bowtie)$

Department of Human Genetics, Hadassah Hebrew

University Hospital, Jerusalem 91120, Israel

e-mail: msagi@hadassah.org.il

T. Perez

Sharett Institute, Hadassah University Hospital, Jerusalem,

Israel 